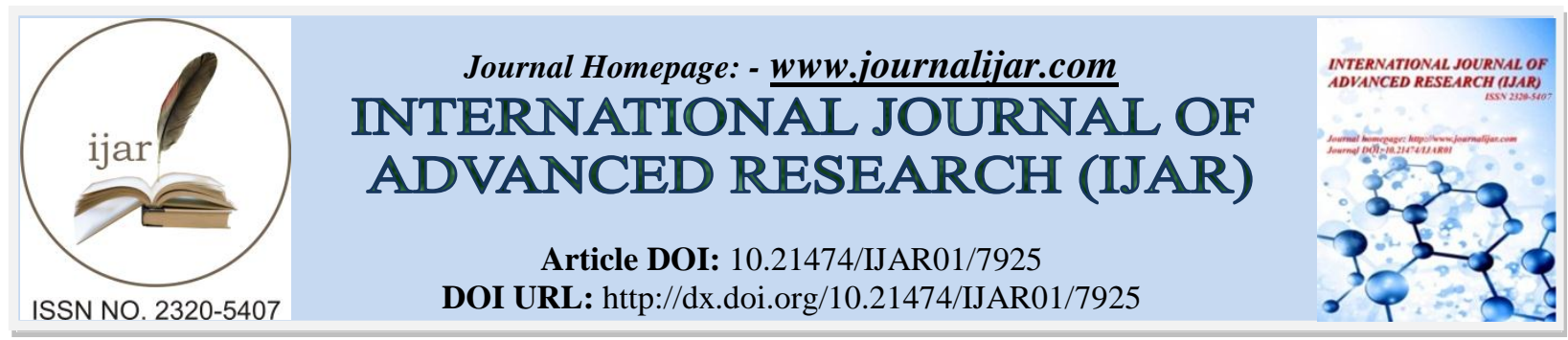

RESEARCH ARTICLE

\title{
CONSOMMATION DE SUBSTANCES PSYCHOACTIVES ET COMPORTEMENTS SEXUELS A RISQUE CHEZ DES POPULATIONS A GUIBEROUA (CENTRE OUEST DE LA COTE D'IVOIRE).
}

\author{
François Kouakou Yao ${ }^{1}$, Abel A. Adjet ${ }^{1}$ and Daniele O. Konan ${ }^{2}$. \\ 1. Enseignant-Chercheur, université Jean Lorougnon Guédé. \\ 2. Doctorante, université Alassane Ouattara.
}

\section{Manuscript Info}

Manuscript History

Received: 14 August 2018

Final Accepted: 16 September 2018

Published: October 2018

Keywords:

Psychoactive Substances-Risky Sexual Behavior- Aphrodisiac Root-Guiberoua

\section{Abstract}

The consumption of psychoactive substances today extends to all socioprofessional groups and has become a real concern for the rulers. His association with sexuality for performance enhancement (sexual) is also more and more described. This paper aims to identify the various risky sexual behaviors associated with the use of alcohol, psychoactive tablets and aphrodisiac roots in Guiberoua populations in west central Côte d'Ivoire. A combination of approach using interview and observation direct indicates that having sex with multiple partners, with unknown partners, without wearing condoms and seeking strong sensation through the number of relationships (ejaculation) of a night are consecutive to the consumption of psychoactive substances.

Copy Right, IJAR, 2018,. All rights reserved.

\section{Introduction:}

L'intérêt, la curiosité pour les plantes médicales ou alimentaires et biens d'autres substances qui ont un effet sur les fonctions sexuelles continuent de retenir l'attention des populations et des chercheurs dans nos sociétés. La palette des remèdes ainsi que leur composition pour la guérison des dysfonctionnements sexuels disponible dans les officines de produits naturels se sont également élargies. (Rowland et Tai, 2003). De nombreux travaux scientifiques ont depuis longtemps porté sur les conséquences de l'usage des drogues, et tout récemment, des médicaments sur les fonctions sexuelles. De ces travaux, différentes dimensions se dégagent. Au premier niveau, des substances aphrodisiaques envisagées sous les angles ethnologiques et expérimentales, il ressort une diversité de contextes et de produits, de même que la variabilité de leur effet sur l'organisme. Le second niveau s'est intéressé aux drogues de synthèse contemporaines et leurs contextes d'utilisation ainsi que leurs conséquences sur la sexualité et les prises de risque face aux maladies sexuellement transmissibles (MST). A un troisième niveau, il est fait mention du développement de la pharmacologie qui module la fonction sexuelle de même que l'usage détourné des médicaments à des fins sexuelles récréatives (Levy et Garnier, 2006). Par ailleurs, des différentes études menées, il ressort que l'utilisation détournée des psychotropes ne date pas de notre ère. En effet, l'évolution industrielle du XVIIIe siècle et le progrès de la chimie au XIXe siècle ont permis d'isoler et de produire des substances destinées au départ à l'usage médical. Mais très vite, leur consommation va être étendue par de nombreuses personnes à la recherche de sensation fortes (Grimal, 1993). L'action supposée ou réelle des plantes et des produits sur le désir, la sensibilité corporelle, l'excitation, le plaisir ou la réduction des inhibitions est ancienne et n'en suscite pas moins l'intérêt de nos contemporains. De même, les médicaments dont on a découvert les effets sur la sexualité ont intégré le lot des drogues illicites récréatives. Ce phénomène a été encore renforcé à partir des années 1980, lorsque la recherche pharmaceutique s'est orientée plus directement vers le traitement des dysfonctions sexuelles masculines 
(Fournie, 2010). Or, dans les sociétés traditionnelles et dans certaines civilisations, des substances psychoactives à l'état naturel n'étaient utilisées que dans des rituels magico-religieux lors de certaines cérémonies. Elles étaient aussi présentes dans le vécu quotidien des populations, mais surtout d'une certaine classe où elles avaient fonction à stimuler la réponse sexuelle lors des relations avec des partenaires. Leur utilisation était basée sur des conceptions médicales fondées sur des savoirs traditionnels et sur des croyances où l'efficacité symbolique côtoie des conceptions plus empiriques (Ratsch, 2001 ; Lemelin, 2012). Dans cette même optique, des études conduites par certains chercheurs (Muller et Ratsch, 1993 ; Cooper, 2002) ont mis en évidence la diversité des substances végétales et animales qui sont considérées comme aphrodisiaques et dont la nomenclature et les usages dépendent des sociétés. Toutefois, les végétaux aux effets érotiques constituent une catégorie privilégiée dans cette liste. Des différentes régions du monde, plus de mille (1000) plantes ont pu être ainsi recensées. Elles sont consommées sous des formes variées ; allant des infusions aux inhalations en passant par des décoctions, plats cuisinés et poudres, afin de stimuler l'appétit ou l'activité sexuelle par leurs effets stimulants. De plus en plus aujourd'hui, ces produits qui ont un effet sur le psychisme et l'organisme du consommateur sont recherchés par les jeunes et jeunes adultes. Comportement que Lariscy, Reber et Paek (2011) ont lié au développement et au visionnage des téléséries. Téléséries dans lesquelles à travers les épisodes et les évènements que vivent les personnages principaux, un ensemble de représentations, de normes et de modèles influencent les conduites des téléspectateurs. Or, dans certains domaines, comme ceux touchant à la sexualité ou à l'usage des drogues et des psychotropes, des sujets qui sont moins abordés avec les parents, les médias figurent parmi les principales sources d'information des jeunes. Pour mieux cerner les représentations de ces produits et des pratiques qui leur sont associées dans les fictions sérielles populaires auprès des jeunes, Sahmsa (2010) propose une analyse de ces productions pour en effet permettre de cerner les personnages, les situations et les comportements qui y sont représentés et aider à saisir les modèles socioculturels auxquels ceux-ci sont confrontés.

Le rapport de l'OMS (2005), tout en révélant le caractère limité de la consommation des psychotropes dans des contextes spécifiques liés à des traditions particulières Jusqu’à une époque récente, reconnait la nouvelle donne mondiale: celle de la plus grande variété de drogues consommées, leur puissance accrue, leurs modes d'administration plus actifs (l'injection par exemple), l'absence d'encadrement social et, partant, un pourcentage plus élevé de consommateurs qui deviennent dépendants, avec les conséquences sanitaires et sociales que cela implique.

En Côte d'Ivoire, la situation de la consommation des substances psychoactives également décrite fait état d'une forte prédominance masculine et d'une forte prédominance du cannabis et des amphétamines (Sangaré et al, 2011 ; Ounnebo, Yao et Kouakou, 2017).

Au-delà de la spécificité du genre dans la consommation et des différents produits couramment utilisés par les adultes, c'est toute la question du mésusage des produits psychotropes par les différentes populations qui se pose. Cette étude se veut une contribution à la recherche sur l'utilisation des produits psychoactifs dans les actes sexuels par la prise en compte des catégories de substances consommées par tranche d'âge.

De ce fait, la question que soulève cette étude est : existe-t-il une relation entre les comportements sexuels à risque et la consommation des substances psychoactives chez des populations à Guiberoua (centre ouest de la Côte d'Ivoire) ?

De cette question découle l'objectif suivant : analyser la relation entre les comportements sexuels à risques chez des populations à Guiberoua et la consommation d'alcool, des comprimés amphétamines et des racines aphrodisiaques. Notre hypothèse est la suivante : les comportements sexuels à risque chez les populations à Guiberoua se justifient par l'usage de l'alcool, des comprimés amphétamines et des racines aphrodisiaques.

L'étude s'inscrit dans le modèle de la théorie de l'addiction rationnelle développée par Becker et Murphy (1988). Ce modèle théorique se propose de fournir une explication des comportements associés aux addictions au moyen d'un modèle économique de choix rationnel. Ainsi, il est démontré à la fois une attente d'une jouissance plus forte après chaque consommation; ce qui est donc appelé «la complémentarité adjacente » et une forte préférence pour l'immédiat pour les sujets addicts.

\section{Methodologie:-}

\section{Terrain et population d'étude}

La commune de Guiberoua qui est située au centre ouest de la Côte d'Ivoire dans la région du Gôh est notre terrain d'étude. Elle est une petite ville du département de Gagnoa située sur l'axe Issia-Gagnoa à 50 kilomètres d'Issia et à 25 kilomètres de Gagnoa avec une superficie de 142.7 hectares et une population de 32.671 habitants (INS-RGPH, 
2014). Guiberoua est une localité où vivent différentes communautés venues d'horizons divers : autochtones Bété, allochtones Baoulé, Sénoufo et allogènes Burkinabés, Maliens et Guinéens.

Le choix de cette localité est justifié par le fait qu'elle est un lieu de rencontre privilégié pour différentes personnes venant à la fois des villes d'Issia et de Gagnoa. La ruée des populations les week-ends pour prendre part aux différentes cérémonies funéraires organisées parfois en grande pompe. Ces moments de rencontres constituent des occasions de consommation de toute sorte d'alcool et/ou de substances psychoactives ainsi que l'adonnation à divers comportements sexuels.

Ainsi, la population d'enquête dont la tranche d'âge est de 15 à 60 ans est constituée de :

1. Hommes d'âge compris entre 26 et 60 ans $(\mathrm{N}=\mathbf{3 0})$,

2. Jeunes hommes d'âge compris entre 15 et 26 ans $(\mathrm{N}=\mathbf{2 0})$,

3. Femmes d'âge compris entre 15 et 60 ans $(\mathrm{N}=\mathbf{2 0})$,

La taille de notre échantillon qui est de 70 individus est choisie sur la base de l'échantillonnage à boule de neige. L'avantage de cette technique est le fait que sur la base d'un noyau d'amis consommateurs d'alcool et de boissons aphrodisiaques, nous avons pu constituer l'échantillon en ajoutant à notre liste d'autres individus à même de nous renseigner sur notre objet d'étude. L'enquête a été menée du 16 mai au 15 juin 2018.

\section{Outils de recueil et d'analyse des données}

Notre travail se voulant descriptif et donc d'ordre qualitatif, nous avons opté pour des outils de collecte des données tels l'entretien individuel semi-directif, l'observation directe et la recherche documentaire. Les entretiens semidirectifs nous ont permis de comprendre les raisons de l'utilisation des produits psychoactifs dans la sexualité, les catégories de substances utilisées et les tranches d'âge concernées. A l'aide d'une grille d'observation, nous avons pu observer les périodes ou moments de grandes consommations et les préférences par tranche d'âge. La recherche documentaire a porté sur des écrits généraux sur la drogue et la sexualité, les fonctions récréatives de la drogue et sur des articles scientifiques abordant la consommation des produits psychédéliques dans le milieu des jeunes, des bisexuels, des transsexuels ainsi que la consommation de substances psychoactives en période festive. L'approche compréhensive a servi de base d'analyse des données recueillies.

\section{Resultats:}

Les résultats portent sur la typologie des substances psychoactives consommées, la typologie des consommateurs et sur la typologie des comportements sexuels à risques en lien avec la consommation des substances psychoactives.

\section{Typologie des substances psychoactives consommées}

Il s'agit ici de déterminer les différentes substances psychoactives consommées par des populations à Guiberoua en rapport avec leurs comportements sexuels à risque.

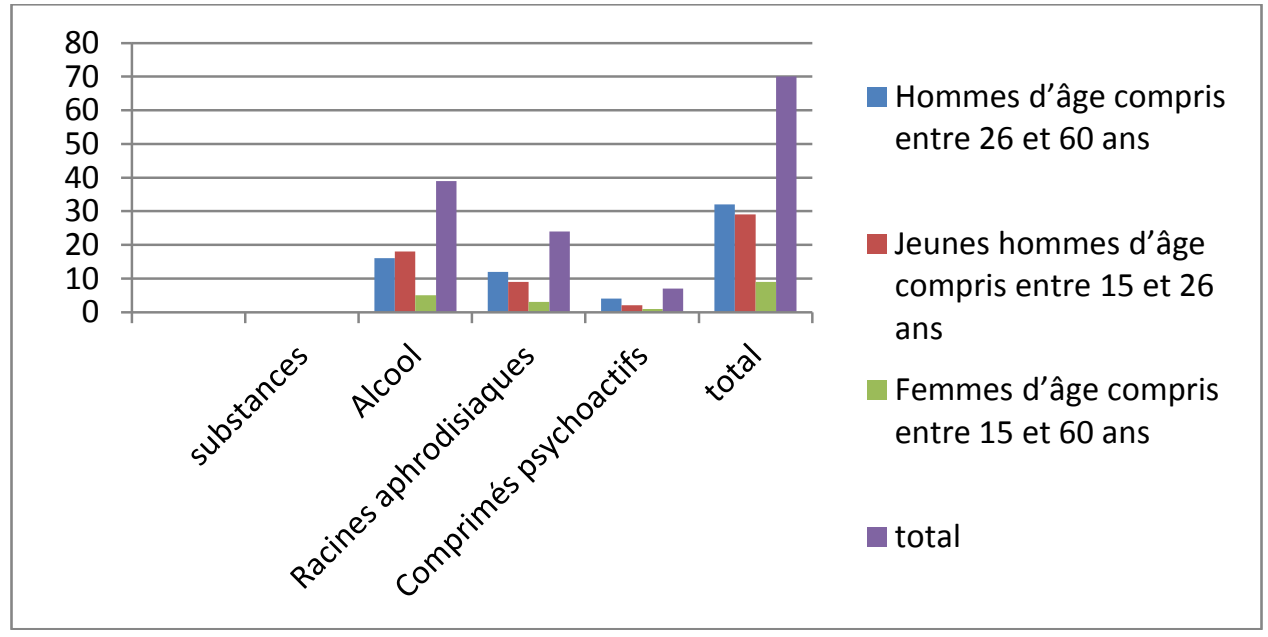

Source : enquête de terrain, mai-juin 2018

Figure 1:-représentation des substances psychoactives consommées selon les enquêtés 
Les substances psychoactives consommées sont l'alcool, les racines aphrodisiaques et les comprimés psychoactifs. Par ailleurs, à l'analyse du graphique (figure 1), il apparait que l'alcool est la première substance psychoactive consommée. Il est suivi des racines aphrodisiaques et des comprimés psychoactifs. Cet état de fait est reconnu autant par les hommes d'âge compris entre 26 et 60 ans, les jeunes hommes d'âge compris entre 15 et 26 ans ainsi que par les femmes d'âge compris entre 15 et 60 ans.

\section{Typologie des consommateurs}

Il s'agit de déterminer les consommateurs des différentes substances psychoactives par tranche d'âge dans l'intervalle $15-60$ ans.

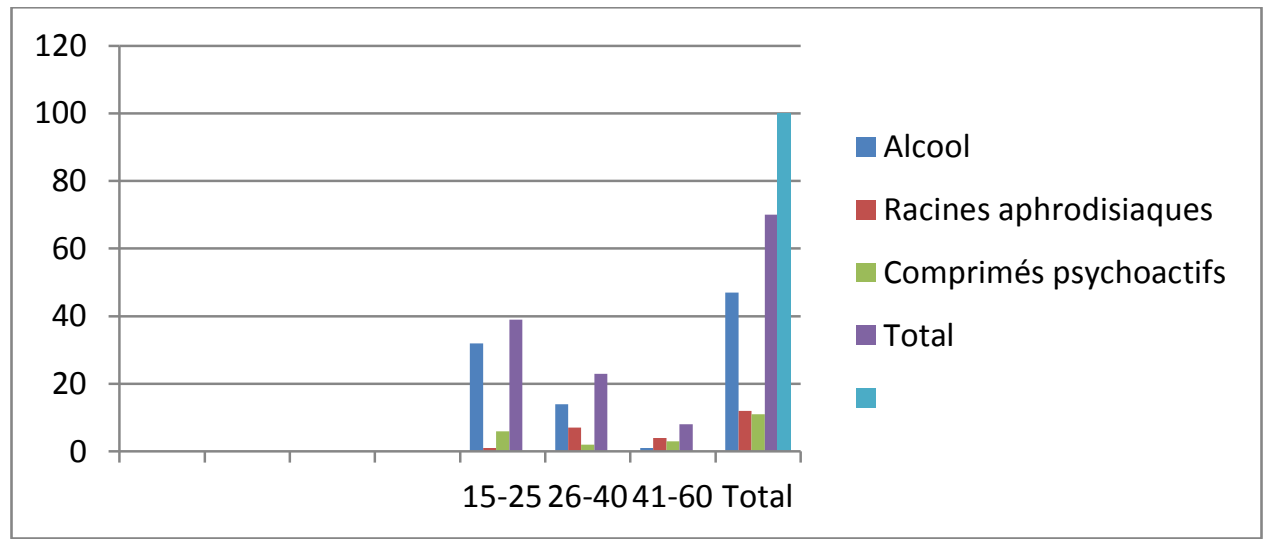

Source : enquête de terrain, mai-juin 2018

Figure 2:-représentation des consommateurs des substances psychoactives par tranche d'âge selon les enquêtés

La tranche d'âge des consommateurs de substances psychoactives en lien avec les comportements sexuels à risque est de 15 à 60 ans. De cette tranche d'âge, une classification est faite en fonction de la substance consommée.

Ainsi, la tranche d'âge 15-25 ans est plus consommatrice d'alcool. Elle est suivie de la tranche d'âge 26-40 ans. La tranche d'âge 41-60 ans est quant à elle plus consommatrice de racines aphrodisiaques (figure 2).

L'analyse du graphique par tranche d'âge révèle que celle de 15 à 25 ans s'intéresse en premier lieu à l'alcool, en deuxième lieu aux comprimés psychoactifs et à un degré moindre aux racines aphrodisiaques.

La tranche d'âge 26-40 ans est plus intéressée d'abord par l'alcool, ensuite par les racines aphrodisiaques et à une faible proportion par les comprimés psychoactifs.

Quant à la tranche d'âge 41-60 ans, en plus d'avoir une consommation des substances psychoactives inférieure aux tranches d'âge précédentes, elle s'illustre d'abord dans la consommation des racines aphrodisiaques, ensuite les comprimés psychoactifs et enfin l'alcool.

\section{Typologie des comportements sexuels à risque}

Il s'agit de déterminer les différents comportements sexuels à risque à la suite de la consommation des substances psychoactives.

Tableau 1:-typologie des comportements sexuels à risque selon les enquêtés

\begin{tabular}{|l|r|r|}
\hline comportements sexuels à risque & nombre cité & Pourcentage \\
\hline rapports sexuels élevés d'une seule nuit & 26 & $37,14 \%$ \\
\hline partenaires multiples & 21 & $30 \%$ \\
\hline partenaires inconnus & 13 & $18,58 \%$ \\
\hline rapports sexuels non protégés & 10 & $14,28 \%$ \\
\hline Total & 70 & $100 \%$ \\
\hline
\end{tabular}

Source : enquête de terrain, mai-juin 2018 
Les comportements sexuels à risque observés chez des populations à Guiberoua en rapport avec la consommation des substances psychoactives sont :

1. Rapports sexuels élevés pour une seule nuit

2. Partenaires multiples

3. Partenaires inconnus

4. Rapports sexuels non protégés

Des comportements sexuels à risque observés, les rapports sexuels élevés durant une seule nuit sont les plus pratiqués avec $26-37.14 \%$ contre $21-30 \%$ pour les partenaires multiples, $13-18.58 \%$ pour les partenaires inconnus et $10-14.28 \%$ pour les rapports sexuels non protégés.

\section{Rapports sexuels élevés d'une seule nuit}

Le premier des comportements sexuels à risque associé à la consommation des substances psychoactives demeure le nombre élevé de rapports sexuels (éjaculation) pour une seule nuit.

En effet, pour prouver sa virilité et conserver l'image de garçon «capable », l'on n'hésite pas à consommer soit de l'alcool, soit des racines aphrodisiaques, soit des comprimés psychoactifs afin de se montrer à la hauteur des attentes de son partenaire sexuel.

Ainsi, cette consommation est dans le seul but d'accroître ou de conserver la puissance sexuelle ou encore de corriger un dysfonctionnement sexuel.

«Un ami qui est venu un jour pour des funérailles à Guiberoua et à qui j'ai trouvé une fille pour son séjour m'a conté qu'il a reçu une gifle matin à son réveil parce qu'il a fait un seul coup (avoir des rapports sexuels une seule fois) et s'est mis à ronfler jusqu'au matin. La fille lui a demandé : c'est pour ça que tu m'as fait venir ? Donc si tu ne veux pas être humilié avec nos filles d'aujourd'hui, il faut assurer en prenant quelque chose pour te renforcer» (K.J cinquantenaire à Guiberoua).

A l'accroissement de la performance sexuelle par la consommation de substances psychoactives, il faut associer la recherche de sensations fortes.

\section{Partenaires multiples}

La multiplication des partenaires sexuels demeure l'un des comportements sexuels à risque consécutif à la consommation de substances psychédéliques. Demeurés insatisfaits avec un seul partenaire sexuel, les consommateurs de produits psychoactifs vont au-delà de la finalité reproductive, récréative et médicale de la fonction sexuelle. Ils sont focalisés uniquement sur la recherche intense et continue du plaisir. A cet effet, toutes leurs rencontres et participation à des activités et festivités se soldent par une nouvelle conquête à visée sexuelle. La consommation de substances psychoactives permet de répondre positivement aux différentes sollicitations des partenaires sexuels puisqu'elles leur procurent l'endurance et la performance sexuelle.

« Je ne bois plus autre boisson à part ma bouteille de racines (racines aphrodisiaques macérées dans de la liqueur) que j'ai fait venir d'Agnibilekro (localité située à l'est de la Côte d'Ivoire). Ces racines, vraiment c'est du bon! Les amis avec qui je suis peuvent en témoigner. Quand je prends ça, je peux gérer (avoir des rapports sexuels) toutes les filles que je veux et je n'ai aucun souci » (H. K. agent à la Sous-préfecture de Guiberoua).

\section{Partenaires inconnus}

L'alcool étant associé aux états de conscience modifié, il désinhibe et réduit les distances sociales. Son association aux racines aphrodisiaques (des racines aphrodisiaques macérées dans des boissons fortes) constitue une porte ouverte à de nombreux vices dont les rapports sexuels avec des partenaires inconnus.

"Quand l'alcool fait son effet au bar ou au maquis, on ne trie plus. Même si tu ne connais pas la fille auparavant et que tu veux (désirer avoir des rapports sexuels) et qu'elle est d'accord (consentante) vous partez. Dans ça on n'a pas besoin de se connaître. On se fait plaisir ». Atteste un jeune homme de 25 ans.

Ainsi, au cours des soirées et rencontres privées où les produits sont utilisés pour décupler le plaisir et le désir, la connaissance préalable du partenaire sexuel devient une insouciance ; d'où les propos de M.H. venu passé le week- 
end: " on n'a pas besoin de connaître la fille. Si elle peut faire l'affaire (répondre aux besoins sexuels), c'est l'essentiel ».

Sur le terrain, les 20 individus de la catégorie de la tranche d'âge 15-25 ans de notre échantillon ont avoué avoir déjà eu des relations sexuelles avec des partenaires qu'ils ne connaissaient pas auparavant; soit $100 \%$ des individus de cette catégorie.

\section{Rapports sexuels non protégés}

Les rapports sexuels non protégés découlent également de la consommation des produits psychédéliques.

Dans un contexte de désinhibition, de sécrétion hormonale, d'excitation sexuelle et d'érectilité accrue où les partenaires sont obnubilés par le désir sexuel après consommation d'un produit psychoactif, il peut être difficile de rechercher un condom pour se protéger. Il s'observe alors une absence de discussion des risques avant les rapports sexuels. Cette absence de discussion débouche sur un accord mutuel tacite pour la non utilisation d'un préservatif. Les risques encourus sont les contractions des Infections Sexuellement Transmissibles (IST), des Maladies Sexuellement Transmissibles (MST), du VIH sida et des grossesses indésirées.

\section{Discussion:-}

La présente étude a porté sur les comportements sexuels à risque chez des populations à Guiberoua en rapport avec la consommation de substances psychoactives. L'objectif est l'identification de leurs comportements sexuels à risque en lien avec la consommation d'alcool, des comprimés psychoactifs et des racines aphrodisiaques. En hypothèse de travail, nous avons retenu que les comportements sexuels à risque chez des populations à Guiberoua se justifient par l'usage de certaines substances psychoactives. En référence aux résultats obtenus, nous pouvons affirmer d'une part que notre objectif est atteint et que ces mêmes résultats valident d'autre part l'hypothèse retenue pour ce travail.

L'association entre la consommation de substances psychoactives, les activités sexuelles et les comportements sexuels à risque est une réalité à Guiberoua.

En effet, comme l'ont reconnu nos enquêtés, la consommation d'alcool, de comprimés psychoactifs et de racines aphrodisiaques induit respectivement une élévation des rapports sexuels durant une seule nuit, une multiplication des partenaires sexuels, des rapports sexuels avec des partenaires inconnus et des rapports sexuels non protégés.

S'agissant des rapports sexuels élevés d'une seule nuit en lien avec la consommation de substances psychoactives, nous pouvons faire remarquer que l'objectif recherché est à la fois la satisfaction du partenaire sexuel et la prouvabilité de sa virilité à travers la recherche de sensations fortes. L'usage d'alcool, de comprimés psychoactifs et de racines aphrodisiaques influe sur les états de conscience érotique et donc favorise une intensification de la réponse sexuelle (Ratsch, 2001 ; Fournie, 2010).

La multiplication des partenaires sexuels en rapport avec la consommation des produits psychédéliques répond à un besoin sans cesse accru de satisfaction de la libido. En conséquence, une diversité de substances considérée comme aphrodisiaque aux effets érotiques est constamment recherché en vue d'une réponse sexuelle positive (Muller et Ratsch ; 1993, Cooper, 2002). Sous ce rapport, les analyses présentées s'inscrivent dans le modèle de la théorie de l'addiction rationnelle de Becker et Murphy (1988). Des individus s'adonnent à la consommation de l'alcool, des comprimés psychoactifs et des racines aphrodisiaques à l'idée d'une potentialisation immédiate de la jouissance sexuelle.

De plus, la consommation de substances psychoactives semble augmenter la tendance naturelle de certaines personnes à rechercher des situations stimulantes et à valoriser davantage les relations de fréquentation de courte durée. Toute chose qui accroît les opportunités de connaître plusieurs partenaires sexuels (Lemelin, 2012).

Par ailleurs, la consommation d'alcool et celle de certaines substances psychoactives dans un contexte plus convivial et plus intime favorise la désinhibition et constitue un prélude aux activités sexuelles avec des partenaires inconnus. Et les rapports sexuels ne sont pas toujours protégés (Sahmsa, 2010). En effet, dans un contexte de "vulnérabilité chimique » où une personne certes, consentante au départ peut, sous l'effet des produits consommés voir son 
consentement modifié ou aboli, sa perception du risque se trouve par ricochet affectée. Ce qui constitue un risque élevé d'infection au VIH sida et autres maladies sexuellement transmissibles.

\section{Conclusion:-}

Cette étude a permis de mettre en évidence le lien entre la consommation de certaines substances psychoactives et les comportements sexuels à risque à Guibéroua en Côte d'Ivoire. La consommation d'alcool, des comprimés psychoactifs et des racines aphrodisiaques conduisent à des rapports sexuels élevés durant une seule nuit, à une multiplication des partenaires sexuels, à des rapports sexuels avec des partenaires inconnus et à des rapports sexuels non protégés. En conséquence et pour une lutte efficace contre la propagation du VIH sida, la relation complexe sexe-drogues et substances psychotropes d'une part et d'autre part, la relation drogue et VIH sida doivent être approfondies.

\section{Références:-}

1. Becker, G. ; Murphy, K. (1988). «A theory of rational addiction », The Journal ofPolitical Economics, 96, pp. 675-700.

2. Cohen, H. et Lévy, J. (1986). Les états modifiés de conscience. Montréal: éditions du Méridien.

3. Cooper, M. (2002). Alcohol use and risky sexual behavior? A complex answer to a simple question. Current direction in psychological science, 15 (1). 19-23.

4. Foucault, M. (1976). Histoire de la sexualité 1. La volonté de savoir. Paris. Editions Gallimard.

5. Garceau-Brodeur, M. (2006). Ecstasy et sexualité: une étude exploratoire au Québec. Drogues, Santé et Société, 5(2), 111-133.

6. Hautefeuille, M. et Véléa, D. (2002). Les drogues de synthèse. Paris. PUF. Que sais-je? Nº3625.

7. Lemelin, C. (2012). Attachement, personnalité, psychopathie, consommation de substances et relations sexuelles chez des adolescents et des jeunes adultes. Thèse de Doctorat. Université du Québec.

8. Levy, J., Garnier, C. (2006). Drogues, médicaments et sexualité. Drogues, santé et société. Vol. 5 n². PP 1148.

9. Levy, J., Garnier, C. et Thoer-Fabre C. (2006). Du viagra à internet : la technologisation de la sexualité. Bulletin d'histoire politique. 15,1, pp 25-36.

10. Levy, J. et Thoer, C. (2008). Usages des médicaments à des fins non médicales chez les adolescents et les jeunes adultes : perspectives empiriques, Drogues santé et société, 7(1), 153-189.

11. Mayet, A. (2012). Étude des trajectoires d'usage de substances psychoactives chez l'adolescent et l'adulte jeune : Exploration de la théorie de l'escalade. Médecine humaine et pathologie. Université René Descartes - Paris V

12. Mueller, C. et Ratsch C. (1993). Le guide mondial des aphrodisiaques. Paris. Manya.

13. Berthier, N. (2016). Les techniques d'enquête en sciences sociales. Paris: Armand Colin.

14. Ounnebo, M., Yao D., Kouakou, F. (2017). Jeunes Migrants Et Consommation De Drogues Dans La Région Du Haut-Sassandra En Côte d'Ivoire (Daloa). European Scientific Journal. Vol.13, No.35 ISSN: 1857 - 7881 (Print) e - ISSN 1857- 7431.

15. Ratsch, C. (2001). Les plantes de l'amour. Les aphrodisiaques de l'antiquité de nos jours. Paris. Lézard.

16. Rowland, D. et Tai, W. (2003). A review of plant-derived and herbal approaches to the treatment of sexual dysfunctions. Journal of sex and marital therapy. 9,3, pp 185-205.

17. Thoër, C., Robitaille, M. (2011). Utiliser des médicaments stimulants pour améliorer sa performance. Usages et discours de jeunes adultes québécois, Drogues santé et société, 10(2), 1-41. 